\title{
On the imbedding of spherically symmetric space times
}

\author{
Dedicated to Professor Y. Akizuki on his 60th birthday \\ By \\ Mineo IkedA, Shin'ichi Kitamura and Makoto Matsumoto
}

(Received July 13, 1963)

\section{\$1. Introduction}

The concept of a spherically symmetric space time is important in the general theory of relativity. Such a space time (abbreviated as s.s. space in this paper) is, mathematically, a Riemannian space whose fundamental form is reducible to

$$
\begin{gathered}
d s^{2}=-A d r^{2}-B\left(d \theta^{2}+\sin ^{2} \theta d \mathcal{P}^{2}\right)+C d t^{2}, \\
B=r^{2} \text { or const. }>0,
\end{gathered}
$$

where $r, \theta, \rho$ and $t$ are spherical polar and temporal coordinates and $A$ and $C$ are positive-valued functions of $r$ and $t$. An s.s. space is denoted by $S_{\mathrm{I}}$ or $S_{\mathrm{II}}$ according to whether $B$ is $r^{2}$ or constant.

Many authors have investigated the geometrical properties of an s.s. space [1], among which the imbedding ${ }^{1)}$ problem was discussed by Eiesland [2] and Takeno [3] along the line of the general theory of Riemannian spaces (see, for example, [4]). For application, however, it will be desirable to obtain a concrete picture of an imbedding. For the Schwarzschild space time, such a picture was used in Fronsdal's physical paper [5], and a related mathematical theory was developed by Fujitani and two of the present authors (M.I. \& M.M.) [6]. This theory is extended to an s.s. space in the present paper.

1) By this we mean, throughout the present paper, the imbedding into a pseudoEuclidean space. 
We now outline our approach. We first consider the 'spatial section' of an s.s. space, that is, the space $V_{3}$ endowed with the fundamental form

$V_{3}$ :

$$
d s^{2}=A_{0} d r^{2}+B\left(d \theta^{2}+\sin ^{2} \theta d \mathcal{P}^{2}\right),
$$

where $A_{0}$ is obtained from $A$ by putting $t=$ const. The imbedding problem of the $V_{3}$ is studied in $\S 2$. On the basis of this result, the imbedding problem of an s.s. space is discussed in $\$ 3$, in which we are led to introduce the space $V_{2}$ with the fundamental form $V_{2}$ :

$$
d s^{2}=(\eta-A) d r^{2}+C d t^{2} .
$$

Here $\eta=1$ or 0 according to whether the s.s. space under consideration is $S_{\mathrm{I}}$ or $S_{\mathrm{II}}$. The imbedding problem for an s.s. space having $A \neq \eta$ is thus reduced to the problem for $V_{3}$ and $V_{2}$. $\S 4$ is devoted to some particular imbeddings for $V_{2}$ which can be expressed in concrete diagrams.

Remarks. We summarize here the known results which have some connection with the present work.

Theorem 0. An s.s. space is at most, of class two, in the sense of imbedding. A necessary and sufficient condition for an s.s. space to be of class one, is given by

$A \neq \eta, \quad 2\left(\ddot{A}-C^{\prime \prime}\right)+\left(\dot{A}^{2}-A^{\prime} C^{\prime}\right) /(\eta-A)+\left(C^{\prime 2}-\dot{A} \dot{C}\right) / C=0$,

where the prime and the dot mean the derivatives with respect to $r$ and $t$ respectively.

It is to be noted that the condition $A \neq \eta$ is meaningless in the case of $S_{\mathrm{II}}$, because $\eta=0$ and $A>0$ hold.

\section{\$2. The imbedding problem for (1.2)}

We begin our discussions with the imbedding problem of the space $V_{3}$ having the fundamental form (1.2).

First of all, denoting the coordinates $r, \theta$ and $\rho$ by $x^{i}, i=1$, 2,3 , we give the surviving components of the Christoffel symbols $\Gamma_{j k}^{i}$ and the curvature tensor $R_{i j k l}$ of $V_{3}$ : 
On the imbedding of spherically symmetric space times

$$
\begin{aligned}
& \mathrm{I}_{11}^{1}=A_{0}^{\prime} / 2 A_{0}, \quad \mathrm{I}_{22}^{1}=-B^{\prime} / 2 A_{0}, \quad \Lambda_{33}^{11}=-B^{\prime} \sin ^{2} \theta / 2 A_{0}, \\
& \Gamma_{12}^{2}=\Gamma_{13}^{3}=B^{\prime} / 2 B, \quad \Gamma_{23}^{3}=\cot \theta, \quad \Gamma_{33}^{2}=-\sin \theta \cos \theta, \\
& R_{1212}=A_{0}^{\prime} B^{\prime} / 4 A_{0}, \quad R_{1313}=R_{1212} \sin ^{2} \theta, \quad R_{2323}=\left(1-\eta A_{0}\right) B \sin ^{2} \theta \text {. }
\end{aligned}
$$

It is clear from the above expressions that $V_{3}$ is flat if and only if $B=r^{2}$ and $A_{0}=1$. In the case of a non-flat $V_{3}$, an example of the imbedding can easily be given, that is, (1.2) may be written as follows :

$$
\left.\begin{array}{l}
d s^{2}=e\left(d z^{0}\right)^{2}+\left(d z^{1}\right)^{2}+\left(d z^{2}\right)^{2}+\left(d z^{3}\right)^{2}, \\
z^{0}=\int \sqrt{s\left(A_{0}-\eta\right)} d r, \quad e=\operatorname{sign}\left(A_{0}-\eta\right), \\
z^{1}=\sqrt{ } \bar{B} \sin \theta \cos \mathcal{P}, \quad z^{2}=\sqrt{B} \sin \theta \sin \mathcal{P}, \quad z^{3}=\sqrt{ } \bar{B} \cos \theta .
\end{array}\right\}
$$

Thus the following result is obtained.

Theorem 1. The space $V_{3}$ with the fundamental form (1.2) is flat if and only if $B=r^{2}$ and $A_{0}=1$. When $V_{3}$ is not flat, it is of class one.

We next consider the equations of Gauss and Codazzi for the non-flat $V_{3}$ :

$$
\begin{gathered}
e^{\prime} R_{i j k l}=b_{i k} b_{j l}-b_{i l} b_{j k}, \\
\nabla_{i} b_{j k}-\nabla_{j} b_{i k}=0,
\end{gathered}
$$

where $e^{\prime}$ is the indicator of the normal in the enveloping pseudoEuclidean space $E_{3}, b_{i j}$ is the second fundamental tensor and $\nabla_{i}$ denotes the covariant derivative. For the solution of (2.2), we may use the result of Thomas [7] concerning Riemannian spaces of class one. From (2.2) we have

$\left[\operatorname{det} .\left(b_{i j}\right)\right]^{2}=e^{\prime} R_{2323} R_{3131} R_{1212}=e^{\prime} A_{0}^{\prime 2} B^{\prime 2} B\left(1-\eta / A_{0}\right) \sin ^{4} \theta / 16 A_{0}^{2}$.

It is known that if this equantity does not vanish, the $V_{3}$ is intrinsically rigid, i.e. $b_{i j}$ can be uniquely determined, apart from the signs, by the Gauss-Codazzi equations. Taking this fact into account, we divide the following discussions into two cases according to whether $A_{0}^{\prime} \neq 0$ and $B^{\prime} \neq 0$ or not.

(i) The case $B=r^{2}$ and $A_{0} \neq$ const. In this case, the solution of (2.2) is given by the following apart from the signs: 
74 Mineo Ikeda, Shin'ichi Kitamura and Makoto Matsumoto

$$
\begin{aligned}
& b_{i i}=R_{i j i j} R_{i k i k}\left(e^{\prime} R_{2323} R_{3131} R_{1212}\right)^{-1 / 2}, \\
& b_{i j}=0, \quad(i, j, k \neq),
\end{aligned}
$$

or in the concrete,

$$
\left.\begin{array}{l}
b_{11}=A_{0}^{\prime} B^{\prime} / 4 A_{0} \sqrt{e B\left(1-\eta / A_{0}\right)}, \quad b_{22}=e \sqrt{e B\left(1-\eta / A_{0}\right)}, \\
b_{33}=b_{22} \sin ^{2} \theta, \quad e=\operatorname{sign}\left(A_{0}-\eta\right) .
\end{array}\right\}
$$

It can be shown by direct calculations that this solution satisfies the Codazzi equations (2.3), too. Thus we have proved the following theorem.

Theorem 2. The space $V_{3}$ with $B=r^{2}$ and $A_{0} \neq$ const. is intrinsically rigid. Its second fundamental tensor is given by (2.4) apart from the signs, and the imbedding functions are given by (2.1) apart from a motion in the enveloping pseudo-Euclidean space $E_{3}$.

(ii) The case $B=$ const. or $A_{0}=$ const. In this case, $R_{2323}$ is the only surviving component of $R_{i j k l}$, and the Gauss equations become

$$
\left.\begin{array}{l}
b_{22} b_{33}-\left(b_{23}\right)^{2}=e^{\prime}\left(1-\eta / A_{0}\right) B \sin ^{2} \theta, \\
b_{11} b_{i j}-b_{1 i} b_{1 j}=0, \quad b_{12} b_{i 3}-b_{13} b_{i 2}=0, \quad(i, j=2,3) .
\end{array}\right\}
$$

If $b_{11} \neq 0$, then from the second equation of (2.5) we have $b_{i j}=$ $b_{1 i} b_{1 j} / b_{11}$, which contradicts with the first equation of (2.5). Thus $b_{11}=0$, and we obtain $b_{12}=b_{13}=0$ from the second equation of (2.5). In view of this result, a part of the Codazzi equations (2.3) becomes $b_{i j}^{\prime}-b_{i j} B^{\prime} / 2 B=0,(i, j=2,3)$. The integration of these leads to

$$
b_{i j}=\sqrt{ } \bar{B} c_{i j}(\theta, \mathcal{P}), \quad(i, j=2,3),
$$

where $c_{i j}$ are functions of $\theta$ and $\mathcal{P}$. Putting these expressions in the first equation of (2.5) and the remaining equations of (2.3), we get

$$
\left.\begin{array}{l}
c_{22} c_{33}-c_{23}^{2}=e^{\prime}\left(1-\eta / A_{0}\right) \sin ^{2} \theta, \\
\frac{\partial c_{22}}{\partial \rho}-\frac{\partial c_{23}}{\partial \theta}-c_{23} \cot \theta=0, \\
\frac{\partial c_{23}}{\partial \rho}-\frac{\partial c_{33}}{\partial \theta}+c_{22} \sin \theta \cos \theta+c_{33} \cot \theta=0 .
\end{array}\right\}
$$


It can be seen that these are nothing but the Gauss-Codazzi equations for a space of constant curvature whose fundamental form is given by

$$
d s^{2}=\left(1-\eta / A_{0}\right)\left(d \theta^{2}+\sin ^{2} \theta d \mathcal{P}^{2}\right) .
$$

Summarizing the above, we obtain the following result.

Theorem 3. The non-flat space $V_{3}$ with $A_{0}=$ const. or $B=$ const. is not intrinsically rigid, and (2.1) gives an example of imbedding functions. The imbedding problem in this case is equivalent to the problem of finding $e^{\prime}$ and $c_{i j}(\theta, \mathcal{P}),(i, j=2,3)$, which satisfy (2.6). This is the imbedding problem for a two-dimensional space of constant curvature.

\section{§3. The imbedding problem of an s.s. space}

In this section, the imbedding problem of an s.s. space is discussed in the light of the foregoing investigation.

Let us consider an s.s. space, which has the fundamental form (1.1). By a suitable coordinate transformation of the type

$$
\bar{r}=r, \quad \bar{\theta}=\theta, \quad \overline{\mathcal{P}}=\mathcal{P}, \quad \bar{t}=\bar{t}(r, t) ; \quad \dot{\bar{t}} \neq 0,
$$

(1. 1) can be reduced to

$$
d s^{2}=\bar{C} d \bar{t}^{2}+2 \bar{D} d \bar{r} d \bar{t}-\bar{A} d \bar{r}^{2}-\bar{B}\left(d \bar{\theta}^{2}+\sin ^{2} \bar{\theta} d \overline{\mathcal{P}}^{2}\right),
$$

where $\bar{A}=\bar{A}(\bar{r}) \neq 1, \bar{B}=\bar{r}^{2}$ or const. and $\bar{C}$ and $\bar{D}$ are functions of $\bar{r}$ and $\bar{t}$. In view of (2.1), (3.1) may be written as

$$
d s^{2}=d \sigma^{2}-\left(d z^{1}\right)^{2}-\left(d z^{2}\right)^{2}-\left(d z^{3}\right)^{2},
$$

where $d z^{i},(i=1,2,3)$, have expressions similar to (2.1) and

$$
d \sigma^{2}=\bar{C} d \bar{t}^{2}+2 \bar{D} d \bar{r} d \bar{t}+(\eta-\bar{A}) d \bar{r}^{2} .
$$

If we perform the transformation inverse to the above, (3.2) must be reduced to (1.3). We have thus arrived at the fundamental form of $V_{2}$. Here it is to be noted that $A$ in (1.3) is a generic positivevalued function of $r$ and $t$.

It is easily seen that, in the case $A \neq \eta$, the s.s. space is of class one when and only when the $V_{2}$ is flat. The condition re- 
quired for $V_{2}$ to be flat can be obtained by calculating the curvature tensor, and the result coincides with the second equation of (1.4). Thus we obtain the following theorem.

Theorem 4. The imbedding problem of an s.s. space with $A=\eta$ is reduced to the problem for $V_{3}$ and $V_{2}$. Such an s.s. space is of class one if and only if the $V_{2}$ is flat.

On the other hand, in the case $A=\eta$, the fundamental form (1.3) or (3.2) is degenerate, and the results of $\S 2$ cannot be employed for the imbedding problem of the s.s. space.

\section{\$4. The imbedding problem for (1.3)}

From the result in the foregoing section, we see that the space $V_{2}$ with the fundamental form (1.3) has a close connection with the imbedding problem of an s.s. space. In this section, we treat the non-flat space $V_{2}$ in the case where $A=A(r)$ and $C=C(r)$, and obtain some particular imbeddings which can be expressed in concrete diagrams.

The equations of Gauss and Codazzi for (1.3) are easily written down as

$$
\begin{aligned}
& \varepsilon\left(b_{00} b_{11}-b_{01}^{2}\right)=-C^{\prime \prime} / 2+A^{\prime} C^{\prime} / 4(A-\eta)+C^{\prime 2} / 4 C, \\
& b_{00}^{\prime}-\dot{b}_{01}-b_{00} C^{\prime} / 2 C+b_{11} C^{\prime} / 2(A-\eta)=0 \\
& b_{01}^{\prime}-\dot{b}_{11}+b_{01}\left\{C^{\prime} / 2 C-A^{\prime} / 2(A-\eta)\right\}=0
\end{aligned}
$$

where the coordinates $t$ and $r$ are denoted by $x^{i},(i=0,1), \varepsilon$ is the indicator of the normal and $b_{i j}$ is the second fundamental tensor. It is difficult to obtain the explicit solutions of the above equations, and we exclusively study particular solutions having the condition $b_{01}=0$. Such solutions may be called stationary after the corresponding solutions in the Schwarzschild space time [6]. Under the assumption $b_{01}=0$, the above equations are easily integrated, and the result is given by the following apart from the signs.

$$
\left.\begin{array}{l}
b_{00}=\sqrt{\varepsilon\left\{\left(C^{\prime 2} / 4(A-\eta)-a C\right\}\right.}, \quad b_{01}=0, \\
b_{11}=\varepsilon\left\{-C^{\prime \prime}+A^{\prime} C^{\prime} / 2(A-\eta)+C^{\prime 2} / 2 C\right\} / 2 b_{00},
\end{array}\right\}
$$

where $a$ is the integration constant and $\varepsilon=\operatorname{sign}\left\{C^{\prime 2} / 4(A-\eta)-a C\right\}$. 
Now, we consider the formulae of Gauss and Weingarten, which take the following form:

$$
\nabla_{i} \nabla_{j} z=\varepsilon b_{i j} n, \quad \nabla_{j} n=-g^{i k} b_{i j} \nabla_{k} z
$$

or in the concrete,

$$
\begin{aligned}
& \ddot{\boldsymbol{z}}=\left\{C^{\prime} / 2(A-\eta)\right\} \boldsymbol{z}^{\prime}+\varepsilon b_{00} \boldsymbol{n}, \quad \dot{\boldsymbol{z}}^{\prime}=\left(C^{\prime} / 2 C\right) \dot{\boldsymbol{z}}, \\
& \boldsymbol{z}^{\prime \prime}=\left\{A^{\prime} / 2(A-\eta)\right\} \boldsymbol{z}^{\prime}+\varepsilon b_{11} \boldsymbol{n}, \\
& \dot{\boldsymbol{n}}=-\left(b_{00} / C\right) \dot{\boldsymbol{z}}, \quad \boldsymbol{n}^{\prime}=\left\{b_{11} /(A-\eta)\right\} \boldsymbol{z}^{\prime} .
\end{aligned}
$$

Here $\boldsymbol{z}$ and $\boldsymbol{n}$ are respectively the position and the normal vectors in the enveloping pseudo-Euclidean space $E_{3}$. They are subject to the further conditions

$$
\left.\begin{array}{l}
\langle\dot{\boldsymbol{z}}, \dot{\boldsymbol{z}}\rangle=C,\left\langle\boldsymbol{z}^{\prime}, \boldsymbol{z}^{\prime}\right\rangle=\eta-A, \quad\langle\boldsymbol{n}, \boldsymbol{n}\rangle=\varepsilon, \\
\left\langle\dot{\boldsymbol{z}}, \boldsymbol{z}^{\prime}\right\rangle=\langle\dot{\boldsymbol{z}}, \boldsymbol{n}\rangle=\left\langle\boldsymbol{z}^{\prime}, \boldsymbol{n}\right\rangle=0,
\end{array}\right\}
$$

where $\langle$,$\rangle stands for the scalar product of vectors.$

Our next task is to give the imbedding functions $z$ explicitly. Integrating the second equation of (4.3), we have

$$
\boldsymbol{z}=\sqrt{\mathrm{C}} \boldsymbol{f}(t)+\boldsymbol{g}(r),
$$

where $\boldsymbol{f}(t)$ and $\boldsymbol{g}(r)$ are vector functions of $t$ and $r$ respectively. On the other hand, if we differentiate the first equation of (4.3) and use the second of (4.3) and the first of (4.4), we obtain

$$
\dddot{\boldsymbol{z}}+\left\{C^{2} / 4 C(\eta-A)+\varepsilon b_{00}^{2} / C\right\} \dot{\boldsymbol{z}}=0 .
$$

The quantity in the brackets is equal to $-a$ according to the first equation of (4.2). Therefore, the following equation for $\boldsymbol{f}$ in (4.6) is obtained:

$$
\dddot{\boldsymbol{f}}-a \dot{\boldsymbol{f}}=0 .
$$

It is convenient to divide the subsequent discussions according to whether $a<0$ or $>0$ or $=0$.

(i) The case $a \equiv-k^{-2}<0$. The integration of (4.7) gives

$$
\boldsymbol{f}=k \sin (t / k) \boldsymbol{e}_{1}+k \cos (t / k) \boldsymbol{e}_{2},
$$

where $\boldsymbol{e}_{1}$ and $\boldsymbol{e}_{2}$ are constant vectors. We have taken the additive 
integration constants equal to zero, because they may be absorbed into $\boldsymbol{g}$ in the equation (4.6). $\boldsymbol{e}_{1}$ and $\boldsymbol{e}_{2}$ satisfy the condition $\left\langle\boldsymbol{e}_{i}, \boldsymbol{e}_{j}\right\rangle=\delta_{i j}, \quad(i, j=1,2)$, according to the first equation of (4.5). Putting (4.6) with (4.8) in the fourth equation of (4.5), we get

$$
\boldsymbol{g}(\boldsymbol{r})=g(\boldsymbol{r}) \boldsymbol{e}_{3},
$$

where $\boldsymbol{e}_{3}$ is a constant vector orthogonal to $\boldsymbol{e}_{1}$ and $\boldsymbol{e}_{2}$. By further putting (4.6) with (4.8) and (4.9) in the second equation of (4.5), we have

$$
g^{\prime 2}=\varepsilon^{\prime}\left(\eta-A+a C^{\prime 2} / 4 C\right), \quad \varepsilon^{\prime}=\operatorname{sign}\left(\eta-A+a C^{\prime 2} / 4 C\right),
$$

and $\left\langle\boldsymbol{e}_{3}, \boldsymbol{e}_{3}\right\rangle=\varepsilon^{\prime}$. If we gather the above results, the imbedding functions in the present case become

$$
\boldsymbol{z}=k \sqrt{ } \bar{C}\left\{\sin (t / k) \boldsymbol{e}_{1}+\cos (t / k) \boldsymbol{e}_{2}\right\}+g(r) \boldsymbol{e}_{3},
$$

where $g(r)$ is subject to the condition (4.10).

(ii) The case $a \equiv k^{-2}>0$. This case may be treated in the same way as in case (i), and the result is as follows:

$$
\boldsymbol{z}=k \sqrt{ } \bar{C}\left\{\sinh (t / k) \boldsymbol{e}_{1}+\cosh (t / k) \boldsymbol{e}_{2}\right\}+g(r) \boldsymbol{e}_{3},
$$

where $g(r)$ satisfies the equation (4.10) and the constant vectors $\boldsymbol{e}_{i}$ are subject to

$$
\begin{array}{ll}
\left\langle\boldsymbol{e}_{1}, \boldsymbol{e}_{1}\right\rangle=-\left\langle\boldsymbol{e}_{2},\right. & \left.\boldsymbol{e}_{2}\right\rangle=1, \quad\left\langle\boldsymbol{e}_{3}, \boldsymbol{e}_{3}\right\rangle=\varepsilon^{\prime}, \\
\left\langle\boldsymbol{e}_{i}, \boldsymbol{e}_{j}\right\rangle=0, \quad(i \neq j) . &
\end{array}
$$

(iii) The case $a \equiv 0$. This case is slightly different from the above two. The integration of (4.7) gives, apart from the additive constants,

$$
\boldsymbol{f}=t^{2} \boldsymbol{a}_{1}+t \boldsymbol{a}_{2},
$$

where $\boldsymbol{a}_{1}$ and $\boldsymbol{a}_{2}$ are constant vectors, which are subject to the following conditions according to the first equation of (4.5):

$$
\left\langle\boldsymbol{a}_{2}, \boldsymbol{a}_{2}\right\rangle=1,\left\langle\boldsymbol{a}_{1}, \boldsymbol{a}_{1}\right\rangle=\left\langle\boldsymbol{a}_{1}, \boldsymbol{a}_{2}\right\rangle=0 .
$$

Here let us introduce a constant vector $\boldsymbol{a}_{3}$ which satisfies

$$
\left\langle\boldsymbol{a}_{1}, \boldsymbol{a}_{3}\right\rangle=1,\left\langle\boldsymbol{a}_{2}, \boldsymbol{a}_{3}\right\rangle=\left\langle\boldsymbol{a}_{3}, \boldsymbol{a}_{3}\right\rangle=0 .
$$


$\boldsymbol{g}(\boldsymbol{r})$ in (4.6) may be expressed linearly in terms of $\boldsymbol{a}_{i}$. Then, from the fourth equation of (4.5) we have

$$
\boldsymbol{g}(\boldsymbol{r})=g_{1}(\boldsymbol{r}) \boldsymbol{a}_{1}-(\sqrt{ } \bar{C} / 2) \boldsymbol{a}_{3},
$$

where we have omitted the additive constants, because they can be removed by a translation in the enveloping space $E_{3}$. According to the second equation of (4.5), $g_{1}$ satisfies

$$
g_{1}^{\prime}=2 \sqrt{ } \bar{C}(A-\eta) / C^{\prime},
$$

where $C^{\prime} \neq 0$ for the space $V_{2}$ under consideration, i.e. the non-flat $V_{2}$ in which $A=A(r)$ and $C=C(r)$ hold. Here introduce the vectors $\boldsymbol{e}_{i}$ defined by

$$
e_{1}=a_{1}+a_{3} / 2, \quad e_{2}=a_{2}, \quad e_{3}=a_{1}-a_{3} / 2,
$$

and they satisfy the conditions

$$
\begin{aligned}
& \left\langle\boldsymbol{e}_{1}, \boldsymbol{e}_{1}\right\rangle=\left\langle\boldsymbol{e}_{2}, \boldsymbol{e}_{2}\right\rangle=-\left\langle\boldsymbol{e}_{3}, \boldsymbol{e}_{3}\right\rangle=1, \\
& \left\langle\boldsymbol{e}_{\boldsymbol{i}}, \boldsymbol{e}_{\boldsymbol{j}}\right\rangle=0, \quad(i \neq j) .
\end{aligned}
$$

Expressing the above result in terms of $\boldsymbol{e}_{i}$, we are led to

$$
z=\left[\left\{\left(t^{2}-1\right) \sqrt{ } \bar{C}+g_{1}(r)\right\} / 2\right] e_{1}+t \sqrt{ } \bar{C} e_{2}+\left[\left\{\left(t^{2}+1\right) \sqrt{C}+g_{1}(r)\right\} / 2\right] e_{3} .
$$

If we summarize the results in this section, the following theorem is obtained.

Theorem 5. The stationary imbedding of $V_{2}$ is given by one of the following:

$$
\begin{aligned}
& \text { (i) } \quad d s^{2}=\left(d z^{4}\right)^{2}+\left(d z^{5}\right)^{2}+\varepsilon^{\prime}\left(d z^{6}\right)^{2}, \\
& z^{4}= k \sqrt{C} \sin (t / k), \quad z^{5}=k \sqrt{C} \cos (t / k), \quad z^{6}=g(r), \\
& \varepsilon^{\prime}= \operatorname{sign}\left(\eta-A-k^{2} C^{\prime 2} / 4 C\right), \quad g^{\prime 2}=\varepsilon^{\prime}\left(\eta-A-k^{2} C^{\prime 2} / 4 C\right), \quad k \neq 0 . \\
& \text { (ii ) } \quad d s^{2}=\left(d z^{4}\right)^{2}-\left(d z^{5}\right)^{2}+\varepsilon^{\prime}\left(d z^{6}\right)^{2}, \\
& z^{4}= k \sqrt{C} \sinh (t / k), \quad z^{5}=k \sqrt{C} \cosh (t / k), \quad z^{6}=g(r), \\
& \varepsilon^{\prime}= \operatorname{sign}\left(\eta-A+k^{2} C^{\prime 2} / 4 C\right), \quad g^{\prime 2}=\varepsilon^{\prime}\left(\eta-A+k^{2} C^{\prime 2} / 4 C\right), \quad k \neq 0 . \\
& \text { (iii) } \quad d s^{2}=\left(d z^{4}\right)^{2}+\left(d z^{5}\right)^{2}-\left(d z^{6}\right)^{2}, \\
& z^{4}=\left\{\sqrt{C}\left(t^{2}-1\right)+g(r)\right\} / 2, \quad z^{5}=\sqrt{C} t, \\
& z^{6}=\left\{\sqrt{ } \bar{C}\left(t^{2}+1\right)+g(r)\right\} / 2, \quad g^{\prime}=2 \sqrt{C}(A-\eta) / C^{\prime} .
\end{aligned}
$$


From this theorem and (2.1), we obtain the imbedding functions for the s.s. space of class two, in which $A=A(r)$ and $C=C(r)$ hold. When the functional forms of $A$ and $C$ are given concretely, this theorem enables us to diagram the surface $V_{2}$ imbedded in a pseudo-Euclidean space $E_{3}$. Such a diagram will be useful in physical applications.

\author{
Research Institute for Theoretical Physics, \\ Hiroshima University; Fukui University \\ and Institute of Mathematics, Kyoto University.
}

\title{
REFERENCES
}

[1] H. Takeno: The theory of spherically symmetric space-times, Sci. Rep. Research Inst. Theor. Phys. Hiroshima Univ. No. 3 (1963), which contains an extensive bibliography.

[2] J. Eiesland: The group of motions of an Einstein space, Trans. Amer. Math. Soc. 27 (1925), 213-245.

[3] H. Takeno: Theory of the spherically symmetric space-times III, Jour. Sci. Hiroshima Univ. A 16 (1952), 291-298.

[4] L. P. Eisenhart: Riemannian geometry, Princeton (1949).

[5] C. Fronsdal : Completion and embedding of the Schwarzschild solution, Phys. Rev. 116 (1959), 778-781.

[6] T. Fujitani, M. Ikeda and M. Matsumoto : On the imbedding of the Schwarzschild space-time II, Jour. Math. Kyoto Univ. 1 (1961), 63-70.

[7] T. Y. Thomas : Riemann spaces of class one and their characterizations, Acta Math. 67 (1936), 169-211. 